\title{
Efficient Modeling of Concurrent Systems in BMC
}

\author{
Malay K. Ganai and Aarti Gupta \\ NEC Labs America, Princeton, NJ, USA
}

\begin{abstract}
We present an efficient method for modeling multi-threaded concurrent systems with shared variables and locks in Bounded Model Checking (BMC), and use it to improve the detection of safety properties such as data races. Previous approaches based on synchronous modeling of interleaving semantics do not scale up well due to the inherent asynchronism in those models. Instead, in our approach, we first create independent (uncoupled) models for each individual thread in the system, then explicitly add additional synchronization variables and constraints, incrementally, and only where such synchronization is needed to guarantee the (chosen) concurrency semantics (based on sequential consistency). We describe our modeling in detail and report verification results to demonstrate the efficacy of our approach on a complex case study.
\end{abstract}

\section{Introduction}

The growth of cheap and ubiquitous multi-processor systems and concurrent library support are making concurrency programming very attractive. On the other hand, verification of concurrent systems remains a daunting task especially due to complex and unexpected interactions between asynchronous threads, and various architecture-specific memory consistency models [1]. In this work, we focus on concurrency semantics based on sequential consistency [2]. In this semantics, the observer has a view of only the local history of the individual threads where the operations respect the program order. Further, all the memory operations exhibit a common total order that respect the program order and has the read value property, i.e., the read of a variable returns the last write on the same variable in that total order. In the presence of synchronization primitives such as locks/unlocks, the concurrency semantics also respects the mutual exclusion of operations that are guarded by matching locks. Sequential consistency is the most commonly used concurrency semantics for software development due to ease of programming, especially to obtain race-free, i.e, correctly synchronized threads. A data race corresponds to a global state where two different threads access the same shared variable, and at least one of them is a write.

Bounded Model Checking (BMC) [3] has been successfully applied to verify realworld designs. Strengths of BMC are manifold: First, expensive quantification used in symbolic model checking [4] is avoided. Second, reachable states are not stored, avoiding blow-up of intermediate state representation. Third, modern SAT solvers are able to search through the relevant paths of the problem even though the paths get longer with the each BMC unrolling. We focus on verifying concurrent systems through efficient modeling in BMC.

\subsection{Related Work}

We discuss various model checking efforts, both explicit and symbolic, for verifying concurrent systems with shared memory. The general problem of verifying a concurrent system with even two threads with unbounded stacks is undecidable [5]. In practice, these verification efforts use incomplete methods, or imprecise models, or sometimes 
both, to address the scalability of the problem. The verification model is typically obtained by composing individual thread models using interleaving semantics, and model checkers are applied to systematically explore the global state space. Model checkers such as Verisoft [6], Zing [7] explore states and transitions of the concurrent system using explicit enumeration. Although several state space reduction techniques based on partial order methods [8] and transactions-based methods [9-12] have been proposed, these techniques do not scale well due to both state explosion and explicit enumeration.

Symbolic model checkers such as BDD-based SMV [4], and SAT-based Bounded Model Checking (BMC) [3] use symbolic representation and traversal of state space, and have been shown to be effective for verifying synchronous hardware designs. There have been some efforts [13-16] to combine symbolic model checking with the above mentioned state-reduction methods for verifying concurrent software. However, they still suffer from lack of scalability. To overcome this limitation, some researchers have employed sound abstraction [7] with bounded number of context switches [17], while some others have used finite-state model $[15,18]$ or Boolean program abstractions with bounded depth analysis [19]. This is also combined with a bounded number of context switches known a priori [15] or a proof-guided method to discover them [18]. To the best of our knowledge, all these model checking methods use synchronous modeling of interleaving semantics. As we see later, our focus is to move away from such synchronous modeling in BMC in order to obtain significant reduction in the size of the BMC instances.

Another development is the growing popularity of Satisfiability-Modulo Theory (SMT)-solvers such as [20]. Due to their support for richer expressive theories beyond Boolean logic, and several latest advancements, SMT-based methods are providing more scalable alternatives than BDD-based or SAT-based methods. In SMT-based $\mathrm{BMC}$, a BMC problem is translated typically into a quantifier-free formula in a decidable subset of first order logic, instead of translating it into a propositional formula, and the formula is then checked for satisfiability using an SMT solver. Specifically, with several acceleration techniques, SMT-based BMC has been shown [21] to scale better than SAT-based BMC for finding bugs.

There have been parallel efforts [22-24] to detect bugs for weaker memory models. As shown in [25], one can check these models using axiomatic memory style specifications combined with constraint solvers. Note, though these methods support various memory models, they check for bugs using given test programs. There has been no effort so far, to our knowledge, to integrate such specifications in a model checking framework that does not require test programs. There have been other efforts using static analysis $[26,27]$ to detect static races. Unlike these methods, our goal is to find true bugs and to not report false warnings.

\subsection{Our Approach: Overview}

We present an efficient modeling for multi-threaded concurrent systems with shared variables and locks in BMC. We consider $\mathrm{C}$ threads under the assumption of a bounded heap and bounded stack. Using this modeling, we augment SMT-based BMC to detect violations of safety properties such as data races. The main novelty of our approach is that it provides a sound and complete modeling with respect to the considered concurrency semantics, without the expensive synchronous modeling of interleaving semantics. Specifically, we do not introduce wait-cycles to model interleaving of the individual threads, and do not model a scheduler explicitly. As we see later, these waitcycles are detrimental to the performance of BMC. Instead, we first create independent (decoupled) individual thread models, and add memory consistency constraints 
lazily, incrementally, and on-the-fly during BMC unrolling to capture the considered concurrency semantics. Our modeling preserves with respect to a property the set of all possible executions up to a bounded depth that satisfy the sequential consistency and synchronization semantics, without requiring an a priori bound on the number of context switches. We have implemented our techniques in a prototype SMT-based BMC framework, and demonstrate its effectiveness through controlled experiments on a complex concurrency benchmark. For experiments, we contrast our lazy modeling approach with an eager modeling [13-16] of the concurrent system, i.e., a monolithic model synchronously composed with interleaving semantics (and possibly, with state-reduction constraints) enforced by an explicit scheduler, capturing all concurrent behaviors of the system eagerly.

Outline: We provide a short background in Section 2; motivation in Section 3; illustrate our basic approach with an example in Section 4; formal description of our modeling in Section 5; correctness theorems and discussion on size complexity in Section 6; BMC size-reduction techniques in Section 7; followed by experiments in Section 8, and conclusions in Section 9.

\section{Preliminaries}

\subsection{Concurrent System: Model and Semantics}

We consider a concurrent system comprising a finite number of deterministic boundedstack threads communicating with shared variables, some of which are used as synchronization objects such as locks. Each thread has a finite set of control states and can be modeled as an extended finite state machine (EFSM). An EFSM model is a 5-tuple $\left(s_{0}, C, I, D, T\right)$ where, $s_{0}$ is an initial state, $C$ is a set of control states (or blocks), $I$ is a set of inputs, $D$ is a set of data state variables (with possibly infinite range), and $T$ is a set of 4-tuple $\left(c, g, u, c^{\prime}\right)$ transitions where $c, c^{\prime} \in C, g$ is a Boolean-valued enabling condition (or guard) on state and input variables, $u$ is an update function on state and input variables.

We define a concurrent system model $\mathcal{C S}$ as a 4-tuple $\left(\mathcal{M}, \mathcal{V}, \mathcal{T}, s_{0}\right)$, where $\mathcal{M}$ denotes a finite set of EFSM models, i.e., $\mathcal{M}=\left\{M_{1}, \cdots, M_{n}\right\}$ with $M_{i}=\left(s_{0 i}, C_{i}, I_{i}, D_{i}\right.$ U $\left.\mathcal{V}, T_{i}\right), \mathcal{V}$ denotes a finite set of shared(or global) variables i.e., $\mathcal{V}=\left\{g_{1}, \cdots, g_{m}\right\}, \mathcal{T}$ denotes a finite set of transitions, i.e., $\mathcal{T}=\bigcup_{i} T_{i}, s_{0}$ denotes the initial global state. Note, for $i \neq j, C_{i} \cap C_{j}=\emptyset, I_{i} \cap I_{j}=\emptyset, D_{i} \cap D_{j}=\emptyset$, and $T_{i} \cap T_{j}=\emptyset$, i.e., except for shared variables $\mathcal{V}$, each $M_{i}$ is disjoint. Let $V L_{i}$ denote a set of tuples values for local data state variables in $D_{i}$, and $V G$ denote a set of tuple values for shared variables in $\mathcal{V}$. A global state $s$ of $\mathcal{C S}$ is a tuple $\left(s_{1}, \cdots, s_{n}, v\right) \in \mathcal{S}=$ $\left(C_{1} \times V L_{1}\right) \cdots \times\left(C_{n} \times V L_{n}\right) \times V G$ where $s_{i} \in C_{i} \times V L_{i}$ and $v \in V G$ denotes the values of the shared global variables. Note, $s_{i}$ denotes the local state tuple $\left(c_{i}, x_{i}\right)$ where $c_{i} \in C_{i}$ represents the local control state, and $x_{i} \in V L_{i}$ represents the local data state. A global transition system for $\mathcal{C S}$ is an interleaved composition of the individual EFSM models, $M_{i}$. Each global transition consists of firing of a local transition $t_{i}=\left(a_{i}, g_{i}, u_{i}, b_{i}\right) \in \mathcal{T}$. In a given global state $s$, the local transition $t_{i}$ of model $M_{i}$ is said to be scheduled if $c_{i}=a_{i}$, where $c_{i}$ is the local control state component of $s_{i}$. Further, if enabling predicate $g_{i}$ evaluates to true in $s$, we say that $t_{i}$ is enabled. Note, in general, more than one local transition of model $M_{i}$ can be scheduled but exactly one of them can be enabled ( $M_{i}$ is a deterministic EFSM). The set of all transitions that are enabled in a state $s$ is denoted by enabled $(s)$.

We can obtain a synchronous execution model for $\mathcal{C S}$ by defining a scheduling function $E: \mathcal{M} \times \mathcal{S} \mapsto\{0,1\}$ such that $t$ is said to be executed at global state $s$, iff 
$t \in \operatorname{enabled}(s) \cap T_{i}$ and $E\left(M_{i}, s\right)=1$. Note, in interleaved semantics, at most one enabled transition can be executed at a global state $s$. In this synchronous execution model, each thread local state $s_{i}$ (with shared access) has a wait-cycle, i.e., a self-loop to allow all possible interleavings.

Semantics of a sequentially consistent memory model $[25,28]$ are as follows:

- Program Order Rule: Shared accesses, i.e. read/write to shared variables, should follow individual program thread semantics.

- Total Order Rule: Shared accesses across all threads should have a total order.

- Read Value Rule: A read access of a shared variable should observe the effect of the last write access to the same variable in the total order.

- Mutual Exclusion Rule: Shared accesses in matched locks/unlock operations should be mutually exclusive.

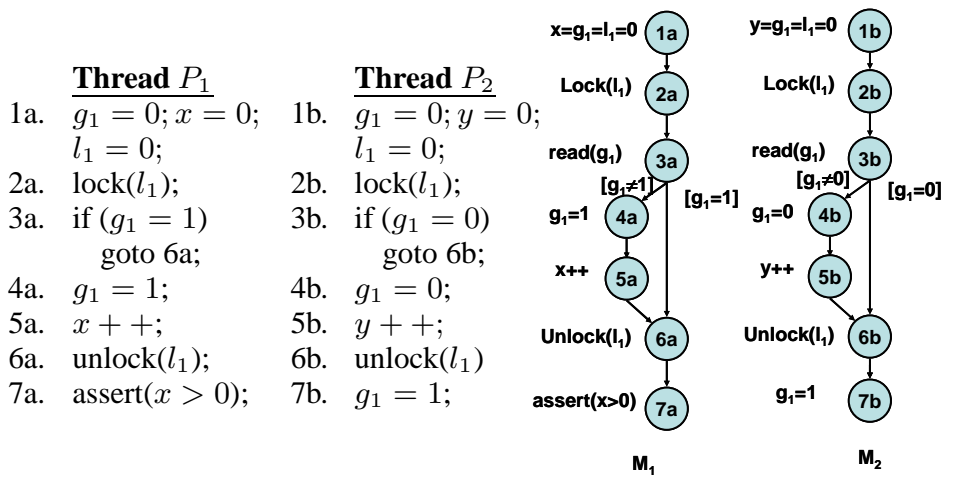

(a)

(b)

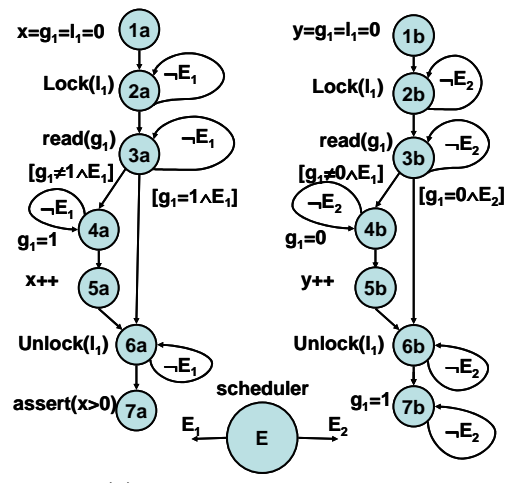

(c)

Fig. 1. (a) Concurrent system with threads $P_{1}$ and $P_{2}$ with local variables $x$ and $y$ respectively, communicating with lock $l_{1}$ and shared variable $g_{1}$. (b) CFG of $P_{1}$ and $P_{2}$, and (c) CFG of concurrent system with scheduler $E$.

Example: We illustrate a concurrent system comprising threads $P_{1}$ and $P_{2}$ with local variables $x$ and $y$, respectively, interacting through lock $l_{1}$ and shared variable $g_{1}$, as shown in Figure 1(a). Each numbered statement is atomic, i.e., it cannot be interrupted. EFSM models $M_{1}$ and $M_{2}$ of the two threads $P_{1}$ and $P_{2}$ are shown as control flow graphs (CFG) in Figure 1(b). Note, $M_{1}$ is the tuple $\left(c_{01}, C_{1}, I_{1}, D_{1}, T_{1}\right)$ with $c_{01}=1 a$, $C_{1}=\{1 a, \cdots, 7 a\}, I_{1}=\{\}, D_{1}=\{x\} \cup\left\{g_{1}, l_{1}\right\}$. The transitions are shown by directed edges with enabling predicates (if not a tautology) shown in square brackets and update functions are shown on the side of each control state. The model $M_{2}$ is similarly defined. An synchronously interleaved model for the concurrent system with threads $P_{1}$ and $P_{2}$, i.e., $\mathcal{C S}=\left(\left\{M_{1}, M_{2}\right\},\left\{g_{1}, l_{1}\right\},\left\{T_{1}, T_{2}\right\},\left((1 a, x),(1 b, y),\left(g_{1}, l_{1}\right)\right)\right)$, with global shared variable $g_{1}$ and lock variable $l_{1}$, and a scheduler $E$ is shown in Figure 1(c). It is obtained by inserting a wait-cycle, i.e., a self-loop at each control state of model $M_{i}$ and associating the edge with a Boolean guard $E_{i}$ such that $E_{i}=1$ iff $E\left(M_{i}, s\right)=1$. To understand the need for such wait-cycles, consider a global state $s$ with thread control states at $2 a$ and $6 b$, respectively. To explore both the interleaving $2 a \rightarrow 3 a$ and $6 b \rightarrow 7 b$ from $s$, each thread needs to wait when the other makes the transition. By noting that the transitions at control states $5 a, 7 a$, and $5 b$ correspond to 
non-shared memory accesses, one can remove the self-loops at these control states. In general, however, all self-loops can not be removed.

\subsection{Building EFSMs from C threads}

For brevity, we highlight the essentials in building a thread model (EFSM) from a C thread (using the F-Soft framework [29]) under the assumption of a bounded heap and a bounded stack. First we obtain a simplified CFG by creating an explicit memory model for (finite) data structures and heap memory, where indirect memory accesses through pointers are converted to direct accesses by using auxiliary variables. We model arrays and pointer arithmetic precisely using a sound pointer analysis. We model loops in the CFG without unrolling them. We handle non-recursive procedures by creating a single copy (i.e., not inlining) and using extra variables to encode the call/return sites. Recursive procedures are inlined up to some user-chosen depth. We perform merging of control nodes in CFG involving parallel assignments to local variables into a basic block, where possible, to reduce the number of such blocks. We, however, keep each shared access as a separate block to allow context-switches.

From the simplified CFG, we build an EFSM with each basic block identified with a unique $i d$ value, and a control state variable $P C$ denoting the current block $i d$. We construct a symbolic transition relation for $P C$, that represent the guarded transitions between the basic blocks. For each data variable, we add an update transition relation based on the expressions assigned to the variable in various basic blocks in the CFG. We use Boolean expressions and arithmetic expressions to represent the guarded and update transition functions, respectively.

\subsection{Control State Reachability (CSR) and CSR-based BMC Simplification}

Control state reachability (CSR) analysis is a breadth-first traversal of the CFG (corresponding to an EFSM model), where a control state $b$ is one step reachable from $a$ iff there is a transition edge $a \longrightarrow b$. At a given sequential depth $d$, let $R(d)$ represent the set of control states that can be reached statically, i.e., ignoring the guards, in one step from the states in $R(d-1)$, with $R(0)=s_{0}$. Computing $C S R$ for the CFG of $M_{1}$ shown in Figure 1(b), we obtain the set $R(d)$ for the first six depths as follows: $R(0)=\{1 a\}, R(1)=\{2 a\}, R(2)=\{3 a\}, R(3)=\{4 a, 6 a\}, R(4)=\{5 a, 7 a\}$, $R(5)=\{6 a\}, R(6)=\{7 a\}$. For some $d$, if $R(d-1) \neq R(d)=R(d+1)$, we say that the $C S R$ saturates at depth $d$, and $R(t)=R(d)$ for $t>d$.

$C S R$ can be used to reduce size of a BMC instance significantly [21]. Basically, if a control state $r \notin R(d)$, then the unrolled transition relation of variables that depend on $r$ can be simplified. We define a Boolean predicate $B_{r} \equiv(P C=r)$, where $P C$ is the program counter that tracks the current control state. Let $v^{d}$ denote the unrolled variable $v$ at depth $d$ during BMC unrolling. Consider the thread model $M_{1}$, where the next state of variable $g_{1}$ is defined as next $\left(g_{1}\right)=B_{1 a}$ ? $0: B_{4 a}$ ? $1: g_{1}$ (using C language notation ?,: for cascaded if-then-else). At depths $k \notin\{0,3\}, B_{1 a}^{k}=B_{4 a}^{k}=0$ since $1 a, 4 a \notin R(k)$. Using this unreachability control state information, we can hash the expression representation for $g_{1}^{k+1}$ to the existing expression $g_{1}^{k}$, i.e., $g_{1}^{k+1}=g_{1}^{k}$. This hashing, i.e., reusing of expressions, considerably reduces the size of the logic formula, i.e., the BMC instance.

\section{Motivation: Why wait-cycles are bad?}

The scope of CSR-based BMC simplification is reduced considerably by a large cardinality of the set $R(d)$, i.e., $|R(d)|$, and hence, the performance of BMC also gets 
affected adversely. In general, re-converging paths of different lengths and different loop lengths are mainly responsible for enlarging set $R$, due to inclusion of all control states in a loop, and ultimately leading to saturation [21]. For example, computing $C S R$ on the concurrent synchronous model (Figure 1(c)), we obtain $R(d)$ as follows:

$R(0)=\{1 a, 1 b\}, R(1)=\{2 a, 2 b\}, R(2)=\{2 a, 3 a, 2 b, 3 b\}, R(3)=\{2 a, 3 a, 4 a, 6 a, 2 b, 3 b, 4 b, 6 b\}$, $R(4)=\{2 a, 3 a, 4 a, 5 a, 6 a, 7 a, 2 b, 3 b, 4 b, 5 b, 6 b, 7 b\}, R(t)=R(4)$ for $t>4$ (Saturates at 4)

Clearly, saturation is inevitable due to the presence of self-loops. At $t \geq 4$, the BMC unrolled transition relation cannot be simplified further using unreachable control states, i.e., not in $R(t)$. Thus, the scope of reusing the expression for next state logic expression is also reduced heavily. In general, saturation can also be caused by program loops. To overcome that we use a Balancing Re-convergence strategy [21] effectively to balance the lengths of the re-convergent paths and loops by inserting NOP states. An NOP state does not change the transition relation of any variable. However, this approach does not work well in the presence of self-loops.

In our experience, synchronous models with self-loops for modeling interleaving semantics are not directly suitable for verifying concurrent systems using BMC. Instead, we propose a modeling paradigm that eliminates self-loops with the goal of reducing the size of BMC instances. However, there are many challenges in doing so in a BMC framework.

- We would like to have soundness and completeness, i.e., neither to miss true witnesses nor to report spurious witnesses (up to some bounded depth). We do so by decoupling the individual thread models, and add the required memory consistency constraints on-the-fly to the unrolled BMC instances. This allows us to exploit advances in SMT-based BMC, without the expensive modeling of interleaving semantics. Note that for bounded analysis the number of shared memory accesses are also bounded, and therefore, these constraints are typically smaller than the model with constraints added eagerly, in practice.

- We also would like to formulate and solve iterative BMC problems incrementally, and integrate seamlessly state-of-the-art advancements in static analysis and BMC. We achieve our goals in a lazy modeling paradigm that simultaneously facilitates the use of several static techniques such as context-sensitive CSR and lockset analysis $[9,10,14]$ and model transformations [21] to accelerate the performance of BMC.

\section{Lazy Modeling Paradigm: Overview}

We illustrate the main idea of our modeling using an example in Section 4.1, and highlight novelties in our approach in Section 4.2. A formal exposition of the modeling and its soundness is provided in Section 5.

\subsection{Basic Approach}

As a first step in our modeling, we construct abstract and independent (i.e. decoupled) thread models $L M_{1}$ and $L M_{2}$ corresponding to the threads $P_{1}$ and $P_{2}$ as shown in Figure 2. We introduce atomic thread-specific procedures read_sync and write_sync before and after every shared access. In Figure 2, the control states $r_{i}$ and $w_{i}$ correspond to calls to procedures read_sync $i$ and write_sync $i$, respectively. We also introduce global variables $T K, C S_{1}$, and $C S_{2}$ described later. For each thread, we make the global variables localized by renaming. As shown in Figure 2, for $P_{1}$ (and similarly for 
$P_{2}$ ), we rename $g_{1}, l_{1}, T K, C S_{1}$, and $C S_{2}$ to local variables $g_{11}, l_{11}, T K_{1}, C S_{11}$, and $C S_{21}$, respectively. The localized shared variables get non-deterministic (ND) values in the control state $r_{i}$. (Refer Section 5.1 for detailed instrumentation.) The models $L M_{i}$ obtained after annotations are independent since the update transition relation for each variable now depends only on the local state variables. Note, there are no self-loops in these models. However, due to ND read values for shared variables in $r_{i}$ control state, these models have additional behaviors, which we eliminate by adding concurrency constraints as described below.

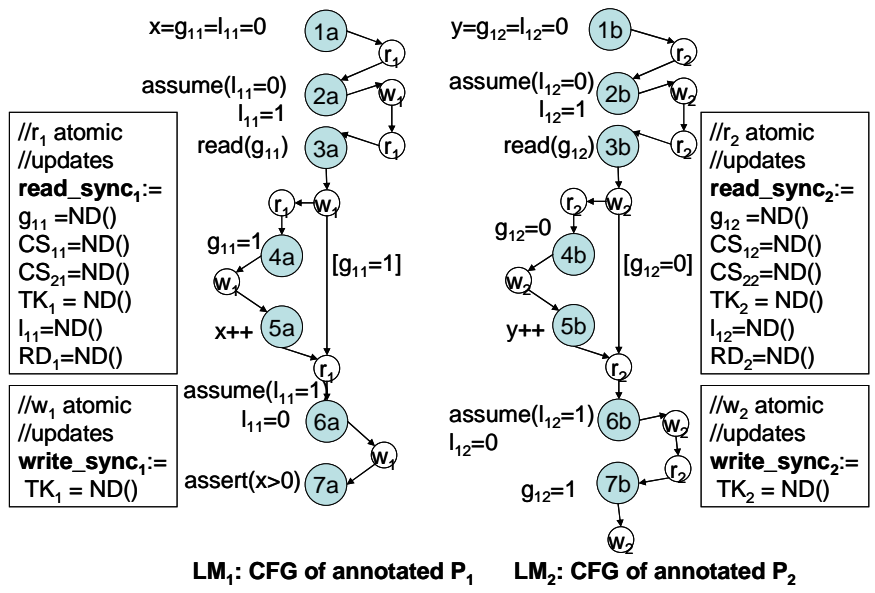

Fig. 2. CFG of threads $P_{1}$ and $P_{2}$ with annotations.

We unroll each model $L M_{i}$ independently during BMC (i.e, with possibly different unroll depths). To each BMC instance, we add concurrency constraints on-the-fly between each pair of control states with accesses to shared variables, that are statically reachable in unrolled CFG at the corresponding thread-specific depths. For sequential consistency, these constraints allow sufficient context-switching to maintain the read value property, and sequentialize the context-switches to enforce a common total order. Note, in addition to these concurrency constraints, a BMC instance comprises transition relation of all thread models and the property constraints. The transition relation of each thread model ensures that memory accesses within the thread follow the program order. Specifically, to capture context-switching events, we added a Boolean shared variable referred to as token $(T K)$. The semantics of a token asserted by a thread is equivalent to a guarantee that all visible operations, i.e., shared memory accesses, issued so far have been committed for other threads to see. Initially, only one thread (chosen nondeterministically) is allowed to assert its token. To track the sequentiality of the global execution and maintain total order, we also add two global clock variables (one per thread) i.e., $C S_{1}$ and $C S_{2}$ to timestamp [28] the token passing events. The pair-wise constraints, added between shared access states, allow passing of the token. Whenever the token is passed from thread $L M_{i}$ (post-access shared state) to $L M_{j}$ (pre-access shared state) the concurrency constraints ensure that each localized shared variable of $L M_{j}$ gets the current state value of the corresponding localized shared variable of $L M_{i}$, and the clock variables get synchronized. We call these pair-wise constraints as ReadWrite Synchronization Constraints as described in Section 5.2 with more details. 


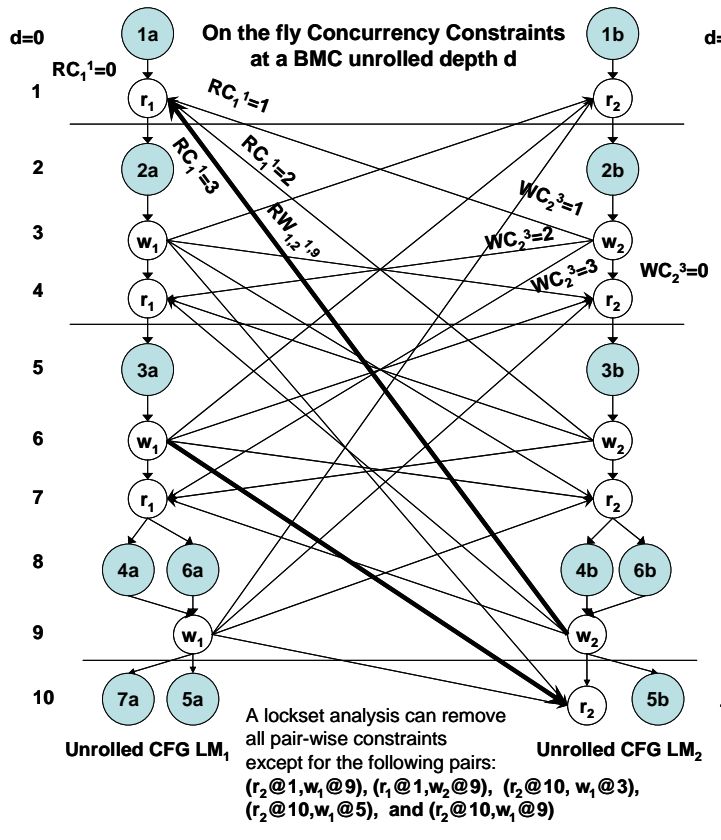

(a)
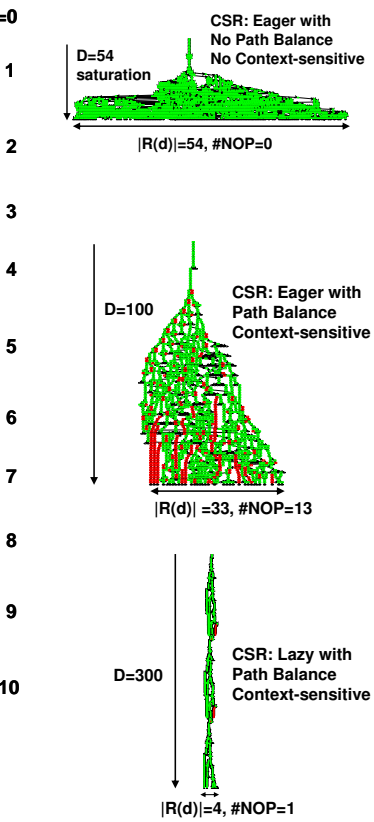

(b)

Fig. 3. (a) Unrolled CFG of thread models $L M_{1}$ and $L M_{2}$ with concurrency constraints added on-the-fly during BMC unrolling. The dark arrows show the token passing events (i.e., context switches) leading to a data race between source lines 3a and 7b (in Figure 1(a)). (b) CSR graphs with path balancing (PB), context-sensitive (CXT) for eager/lazy on a thread-model (Green/Red dots denote non-NOP/NOP blocks, resp.)

We illustrate the concurrency constraints added in Figure 3(a). Let $c @ k$ denote the control state $c$ reached statically at depth $k$. Computing CSR, we obtain $R(0)=$ $\{1 a @ 0,1 b @ 0\}, R(1)=\left\{r_{1} @ 1, r_{2} @ 1\right\}, R(2)=\{2 a @ 2,2 b @ 2\}$ and so on. The concurrency constraints added between a pair $\left(r_{i} @ k, w_{j} @ h\right)$ are shown as an arrow from $w_{j}$ (of $L M_{j}$ ) at depth $h$ to $r_{i}$ (of $L M_{i}$ ) at depth $k$. Note, $r_{i}$ corresponds to pre-access shared state of $L M_{i}$, and $w_{j}$ corresponds to post-access shared state of $L M_{j}$. These constraints also capture the exclusivity of the pair, i.e., constraint for pair $\left(r_{i} @ k, w_{j} @ h\right)$, excludes other pairs $\left(r_{i} @ k, *\right)$ and $\left(*, w_{j} @ h\right)$. For BMC at depth $d<3$ we do not add any pairwise constraints. For BMC at $d=3$, we add concurrency constraints corresponding to pairs $\left(r_{1} @ 1, w_{2} @ 3\right)$ and $\left(r_{2} @ 1, w_{1} @ 3\right)$. For BMC at $d=4$, we add concurrency constraints corresponding to pairs $\left(r_{1} @ 1, w_{2} @ 3\right),\left(r_{2} @ 1, w_{1} @ 3\right),\left(r_{1} @ 4, w_{2} @ 3\right)$, and $\left(r_{2} @ 4, w_{1} @ 3\right)$. Note, in incremental formulation of BMC, we only need to add the constraints corresponding to the last two pairs. In general, the constraints grow quadratically with the analysis depth (ref. Section 6). In addition, we use various static analyses and model transformations to reduce the set of pair-wise constraints added that are redundant (ref. Section 7). For example, using the lockset analysis [9,10,14], we can remove constraints for all pairs other than $\left(r_{2} @ 1, w_{1} @ 9\right),\left(r_{1} @ 1, w_{2} @ 9\right),\left(r_{2} @ 10, w_{1} @ 3\right)$, 
$\left(r_{2} @ 10, w_{1} @ 5\right)$ and $\left(r_{2} @ 10, w_{1} @ 9\right)$. This is because other pairs are not reachable simultaneously in the mutually exclusive region, protected by the lock.

In our approach, a data race condition is detected, if there exists a witness trace where a token passing event occurs between the pair $\left(r_{i} @ k, w_{j} @ h\right)$ with shared accesses on the same variable, with at least one access being a write. In Figure 3(a), we indicate the witness trace corresponding to the data race between source lines $3 \mathrm{a}$ and $7 \mathrm{~b}$ (from Figure 1(c)) as a sequence of the token passing events highlighted in bold arrows between the pairs $\left(r_{1} @ 1, w_{2} @ 9\right)$ and $\left(r_{2} @ 10, w_{1} @ 5\right)$. Note, the control state pair $\left(r_{2} @ 10, w_{1} @ 5\right)$ corresponds to simultaneous accesses of shared variable $g_{1}$ with $r_{2} @ 10$ being a pre-write access state. We obtain the witness trace by unrolling each model in BMC up to depth 11 . In a synchronously interleaved model (i.e., with wait-cycles), we would have obtained the trace at an unrolled depth of $11+7=18$. Note, we sum the two depths, as thread $P_{2}$ has to wait (self-loop) after context switch to $P_{1}$.

For a thread model (example described in Section 8), we compare the reachability graphs on the lazy and eager models, as shown in Figure 3(b), with and without using model transformation such as path/loop balancing (PB) [21] and context-sensitive CSR (CXT) [30]. Note, the width of the graph is proportional to $|R(d)|$. It is desirable that the width is small for greater BMC simplification. We observe that path/loop balancing is more effective on our lazy models (i.e., without wait-cycles) as $R(d)$ is reduced significantly compared to eager models (i.e., with wait-cycles).

\subsection{Our Contributions}

The main idea of our modeling paradigm for concurrent systems is to move away from expensive modeling based on synchronous interleaving semantics. We focus primarily on reducing the size of the BMC problem instances to enable deeper search within the limited resources, both time and memory. Features and merits of our approach are:

1. Lazy modeling constraints: By adding the constraints lazily, i.e., as needed for a bounded depth analysis, as opposed to adding them eagerly, we reduce the BMC problem size at that depth. The size of these concurrency-modeling constraints depends quadratically on the number of shared memory accesses at any given BMC depth in the worst case. Since the analysis depth of BMC bounds the number of shared memory accesses, these constraints are typically smaller than the model with constraints added eagerly, in practice.

2. No wait-cycle: We do not allow local wait cycles, i.e., there are no self-loops in $\mathrm{read} /$ write blocks with shared accesses. This enables us to obtain a reduced set of statically reachable blocks at a given BMC depth $d$, which dramatically reduces the set of pair-wise concurrency constraints that we need to add to the BMC problem.

3. Deeper analysis: For a given BMC depth $D$ and $n$ concurrent threads, we guarantee finding a witness trace (if it exists), i.e., a sequence of global interleaved transitions, of length $\leq n \cdot D$, where the number of local thread transitions is at most $D$. In contrast, an eager modeling approach using BMC [14], an unrolling depth of $n \cdot D$ is needed for such a guarantee. Thus, we gain in memory use by a factor of $n$.

4. Using static analysis: We use property preserving model transformations such as path/loop balancing, and context-sensitive control state reachability to reduce the set of blocks that are statically reachable at a given depth. Again, this potentially reduces the lazy modeling constraints. We also use lockset $[9,10,14]$ analysis to reduce the set of constraints, by statically identifying which block pairs (with shared accesses) are simultaneously unreachable. 
5. SMT-based BMC: We use an SMT solver instead of a traditional SAT solver, to exploit the richer expressiveness, in contrast to bit-blasting. We effectively capture the exclusivity of the pair-wise constraints, i.e., for a chosen shared access pair, other pairs with a common access are implied invalid immediately.

\section{Lazy Modeling of Concurrent Systems}

We present details of our modeling in Sections 5.1 and 5.2.

\subsection{Sound Abstraction: Independent Thread Models}

We perform source-to-source transformations to directly use a model builder (F-Soft [29]) for sequential programs and obtain sound abstraction.

Token: We introduce a global Boolean variable, a token $T K$, to signify that the thread with the token can execute a shared access operation and commit its current shared state to be visible to the future transitions. Initially, only one thread, chosen non-deterministically, is allowed to assert $T K$. Later, this token is passed, from one thread to another, i.e., deasserted in one thread and asserted by the other thread, respectively.

Logical Clock: To obtain a total ordering on token passing events, we use the concept of logical clocks and timestamp [28]. We add a global clock variable $C S_{i}$ for each thread $P_{i}$, so that the tuple $\left(C S_{1} \cdots C S_{n}\right)$ represents the logical clock. These variables are initialized to 0 . Whenever a token $T K$ is acquired by a thread $P_{i}, C S_{i}$ is incremented by 1 in $P_{i}$. The variable $C S_{i}$ keeps track of the number of occurrences of token passing events wherein thread $P_{i}$ acquires the token from another thread $P_{j}, j \neq i$.

Race Detector: We add a race detector local Boolean variable $R D_{i}$ for each thread $P_{i}$. This variable is set to 1 (initially, 0 ) whenever a shared variable is accessed in thread $P_{i}$ and written in another thread $P_{j}$ while token is passed from $P_{j}$ to $P_{i}$.

Localization: For each thread, we make the global variables localized by renaming. Atomic Procedures: We add atomic thread-specific procedures read_sync and write_sync before and after every shared access. In the read_sync procedure, each localized shared and race detector variable get a non-deterministic value, (ND), while in the write_sync procedure only $T K$ gets an ND value.

Synchronization primitives: Operations lock $(l k)$ and unlock $(l k)$ are modeled as atomic operations as sume $(l k=1)$ and as sume $(l k=0)$, respectively. To maintain synchronization semantics, we only consider wait-free execution [15] where the acquisition of the same lock twice is disallowed in a row without an intermediate unlock. Note, this consideration is sufficient to find all data races.

\subsection{Concurrency Constraints}

Given independent abstract models, obtained as above, we add concurrency constraints incrementally, and on-the-fly to each BMC instance, in addition to the transition constraints of the individual thread models and property constraints. The concurrency constraints capture inter- and intra- thread dependencies due to interleavings, and thereby, eliminate additional behaviors in the models up to a bounded depth. Specifically, these constraints comprise (a) pair-wise, i.e., inter-model constraints (shown in Table 1), (b) single-threaded, i.e., intra-model constraints (shown in Table 2), and (c) global constraints (shown in Table 3 ). In the following, we use $R_{i}(d), 0 \leq d \leq D$, to denote the set of control states reachable at depth $d$ for each thread model $L M_{i}$, for a given BMC bound $D$. (Note, we compute CSR on each of the models $L M_{i}$ separately before starting BMC.) Also, we use $x_{i}^{k}$ to denote the expression for the variable $x$ in the unrolled model $L M_{i}$ at depth $k$. 
Table 1. Pair-wise concurrency constraints added in each BMC instance

P1. Read-Write Synchronization Enabling Constraint: For every pair of read_sync control state in $L M_{i}, r_{i} \in R_{i}(k)$ and write_sync control state in $L M_{j}, j \neq i, w_{j} \in R_{j}(h)$, we introduce a Boolean variable $R W_{i j}^{k h}$, and add the following enabling constraint:

$$
R W_{i j}^{k h} \Longleftrightarrow\left(B_{r_{i}}^{k} \wedge \neg T K_{i}^{k} \wedge B_{w_{j}}^{h} \wedge T K_{j}^{h} \wedge C S_{i i}^{k}=C S_{i j}^{h}\right)
$$

If $R W_{i j}^{k h}=1$, we say, the token passing condition is enabled.

P2. Read-Write Synchronization Exclusivity Constraint: Let $R S_{i}^{k}$ define the set $\left\{R W_{i j}^{k h} \mid i \neq\right.$ $j, 0 \leq h \leq d\}$ for a read_sync control state of $L M_{i}$ at depth $k$. To allow at most one write_sync (from a different thread) to match with this read_sync, we assign a unique id $a_{j}^{h} \neq 0$ to each element of $R S_{i}^{k}$. We add a new variable $R C_{i}^{k}$ for the read_sync control state of $L M_{i}$ at depth $k$, require that it takes value $a_{j}^{h} \neq 0$ iff $R W_{i j}^{k h}=1$. Similarly, we introduce a new variable $W C_{j}^{h}$ for the writessync control state of $L M_{j}$ at depth $h$, and require that it takes value $b_{i}^{k} \neq 0$ iff $R W_{i j}^{k h}=1$. The constraints added are:

$$
\begin{aligned}
& R W_{i j}^{k h} \Longleftrightarrow\left(R C_{i}^{k}=a_{j}^{h}\right), a_{j}^{h} \neq 0 \\
& R W_{i j}^{k h} \Longleftrightarrow\left(W C_{j}^{h}=b_{i}^{k}\right), b_{i}^{k} \neq 0
\end{aligned}
$$

Thus, if $R W_{i j}^{k h}=1$, we require that both $R C_{i}^{k} \neq 0$ and $W C_{j}^{h} \neq 0$; and vice-versa.

P3. Read-Write Synchronization Update Constraint: For every $R W_{i j}^{k h}$ variable introduced, we add the following update constraints:

$$
\begin{gathered}
R W_{i j}^{k h} \Longrightarrow \bigwedge_{p=1}^{m} g_{p i}^{k+1}=g_{p j}^{h} \\
R W_{i j}^{k h} \Longrightarrow\left(T K_{i}^{k+1} \wedge \neg T K_{j}^{h+1}\right) \\
R W_{i j}^{k h} \Longrightarrow\left(C S_{i i}^{k+1}=C S_{i i}^{k}+1\right) \wedge\left(\bigwedge_{q=1, q \neq i}^{n} C S_{q i}^{k+1}=C S_{q j}^{h}\right)
\end{gathered}
$$

P4. Data Race Detection Property Constraint: We define two predicates will_access and just_written statically, where will_access $\left(r_{i}, g\right)=1$ iff shared variable $g$ is accessed in the next local control state reachable from $r_{i}$, and just_written $\left(w_{i}, g\right)=1$ iff shared variable $g$ was written in the previous local control state reachable to $w_{i}$. We add the following race detection constraint only if will_access $\left(r_{i}, g\right)=1$ and just_written $\left(w_{j}, g\right)=1$ :

$$
R W_{i j}^{k h} \Longrightarrow R D_{i}^{k+1}
$$


Table 2. Single threaded concurrency constraints added in each BMC instance

S1. No Sync Update Constraint: When none of the token passing events is triggered for a read_sync control state of $L M_{i}$ at depth $k$, we force the next state values to be unchanged for each localized shared and race detector variable in $L M_{i}$ by adding:

$$
\begin{gathered}
R C_{i}^{k}=0 \Longrightarrow\left(\bigwedge_{p=1}^{m} g_{p i}^{k+1}=g_{p i}^{k}\right) \wedge\left(R D_{i}^{k+1}=R D_{i}^{k}\right) \\
R C_{i}^{k}=0 \Longrightarrow T K_{i}^{k+1}=T K_{i}^{k} \\
R C_{i}^{k}=0 \Longrightarrow \bigwedge_{q=1}^{n} C S_{q i}^{k+1}=C S_{q i}^{k}
\end{gathered}
$$

Similarly, for every write_sync control state of $L M_{j}$ at depth $h$, we force the next state token value to be unchanged by adding a similar constraint:

$$
W C_{j}^{h}=0 \Longrightarrow T K_{j}^{h+1}=T K_{j}^{h}
$$

S2. Lock/Unlock Synchronization Constraint: To model assume $(l k=0)$ in lock control state $l_{i}$ of $L M_{i}$ at depth $k$, and similarly, for unlock control state $u l_{i}$, we add

$$
B_{l_{i}}^{k} \Longrightarrow\left(\neg l k_{i}^{k}\right) ; \quad B_{u l_{i}}^{k} \Longrightarrow\left(l k_{i}^{k}\right)
$$

S3. Write Commit Constraint: We make only a write operation commit its current shared state to be visible to the future transitions by adding the following constraint in write_sync control state $w_{j}$ of $L M_{j}$ at depth $h$ corresponding to write operation only, i.e.,

$$
B_{w_{j}}^{h} \Longrightarrow T K_{j}^{h}
$$

S4. Single Control State Reachability Property Constraint: For checking reachability of a local control state $a \in C_{i}$, we add constraint:

$$
B_{a}^{k} \Longrightarrow T K_{i}^{k}
$$

Table 3. Global concurrency constraints added in each BMC instance Single Token Constraint: Initially, exactly one thread model has the token. We add,

$$
\left(\bigvee_{1 \leq i \leq n} T K_{i}^{0}\right) \wedge\left(\bigwedge_{i \neq j} T K_{i}^{0} \Longrightarrow \neg T K_{j}^{0}\right)
$$

Multiple Race Detections: To check multiple data races incrementally, we add the following blocking clause corresponding to the token passing events seen in the last witness trace.

$$
\neg\left(R W_{i j}^{k h} \wedge \cdots \wedge R W_{i^{\prime} j^{\prime}}^{k^{\prime} h^{\prime}}\right)
$$


We add the following pair-wise (i.e., inter-model) constraints P1-P4 between unrolled models $L M_{i}$ and $L M_{j}$ at depths $k$ and $h$, respectively as shown in Table 1.

P1. Read-Write Synchronization Enabling Constraint: The constraints (Eqn 1) capture the enabling of token passing condition. This happens exactly when: a) thread model $L M_{i}$ is in read_sync control state (i.e., pre-access shared state) at depth $k$ and does not hold the token , b) thread model $L M_{j}$ is in write_sync control state (i.e., post-access shared state) at depth $h$ and holds the token, and c) thread model $L M_{j}$ has the latest value of clock variable of $L M_{i}$, and both threads agree on that. Note, this constraint per se is not enough for token passing, and we require the following exclusivity constraint as well.

P2. Read-Write Synchronization Exclusivity Constraint: Exclusivity constraints (Eqn 23 ) ensure that for a chosen pair for token passing $\left(r_{i} @ k, w_{j} @ h\right)$ with $i \neq j$, other pairs $\left(r_{i} @ k, w_{j^{\prime}} @ h^{\prime}\right)$ with $h \neq h^{\prime}$ or $j \neq j^{\prime}$ and $\left(r_{i^{\prime}}^{k^{\prime}}, w_{j}^{h}\right)$ with $i \neq i^{\prime}$ or $k \neq k^{\prime}$ are implied invalid. As shown in Figure 3(a), the pair $\left(r_{1} @ 1, w_{2} @ 3\right)$ excludes other pairs $\left(r_{1} @ 1, w_{2} @ 6\right),\left(r_{1} @ 1, w_{2} @ 9\right),\left(r_{1} @ 4, w_{2} @ 3\right)$, and $\left(r_{1} @ 7, w_{2} @ 3\right)$ due to specific values of variables $R C_{1}^{1}$ and $W C_{2}^{3}$ chosen. Note, Eqn 1, together with Eqn 2 and 3, define $R W_{i j}^{k h}$. We say a token passing event is triggered iff $R W_{i j}^{k h}=1$.

P3. Read-Write Synchronization Update Constraint: If the token passing event is triggered, each localized shared variable of $L M_{i}$ at depth $k$ gets the current state value of the corresponding localized shared variable of $L M_{j}$ at depth $h$ (Eqn 4), the next state value of token of $L M_{i}$ is constrained to 1 , while it is constrained to 0 for $L M_{j}$, indicating a transfer of the token (Eqn 5), the next state value of the clock variable of $L M_{i}$ is incremented by 1 , while the remaining clock variables are sync-ed with that of $L M_{j}$ (Eqn 6).

P4. Data Race or Pair-wise Reachability Property Constraints: A data race is detected, i.e., $R D_{i}^{k+1}=1$ at thread model $i$ at depth $k+1$ whenever for a shared variable $g$ read-write synchronization enabling constraint $R W_{i j}^{k h}$ holds (Eqn 7) with at least one write access on $g$. To check whether control states $a \in C_{i}$ (of $L M_{i}$ ) and $b \in C_{j}$ (of $L M_{j}$ ) are reachable simultaneously, we reduce the reachability problem to a token passing event detection, i.e., $R W_{i j}^{k h}=1$ by adding control states read_sync and write_sync before and after control states $a$ and $b$.

We add following single-threaded (i.e., intra-model) constraints $S 1$-S4 for each thread model $L M_{i}\left(L M_{j}\right)$ at depth $k(h)$ as shown in Table 2.

S1. No Sync Update Constraint: The constraints (Eqn 8-11) keep the states of threadspecific localized global variables, and newly introduced variables unchanged when there is no token passing event (i.e. no context switching) occurs.

S2. Lock/Unlock Synchronization Constraint: The constraints (Eqn 12) assert/deassert the locking predicate variables in the respective lock/unlock states.

S3. Write Commit Constraint: The constraints (Eqn 13) make the write operation of a thread visible to others by asserting the token.

S4. Single Control State Reachability Property Constraint: Like S3, the constraints (Eqn 14) make the reachability of control state visible by asserting the token. 
As shown in Table 3, we add a single token constraint (Eqn 15) needed for total order and blocking clauses (Eqn 16) to detect multiple data races.

\section{Correctness and Size Complexity}

Theorem 1 (Correctness) (A) The lazy modeling constraints allow only those traces that respect the sequential consistency of memory model and synchronization semantics up to the bound D, i.e., our modeling is complete.

(B) Further, if there exists a witness for a reachability property, such that the global trace length is $\leq n \cdot D$ and each local trace length $\leq D$, there exists an equivalent trace allowed by our model corresponding to the witness trace. In other words, our modeling is sound in that it does not miss any witness up to these bounds.

Proof: Here is an outline with details in Appendix A.

- Completeness: Our modeling captures the requirements for sequential consistency (a) program order: using the transition model of each thread, No Sync Update Constraint (Eqn 9-11), and Write Commit Constraint (Eqn 13), (b) total order of shared accesses: using logical clock along with Read-Write Synchronization (Eqn 5 and 6) and No Sync Update Constraint (Eqn 9 and 10), (c) read value rule: using Read-Write Synchronization Constraint (Eqn 4), and No Sync Update Constraint (Eqn 8), and (d) mutual exclusion rule: using Lock/Unlock Synchronization Constraint (Eqn 12).

- Soundness: We add pair-wise constraints for all pairs of shared accesses that are statically reachable up to the bounded depth. Thus, we capture all possible interleavings of shared accesses up to the bound, and hence, we cannot miss any witness up to the bound.

\subsection{Size Complexity}

We now discuss the size of constraints and variables incrementally added at each depth $d$ for $n$ concurrent threads. We consider thread specific read_sync and write_sync procedure calls, without inlining. This implies that at each unrolled depth $k$ of $L M_{i}$, at most one read_sync control state $r_{i}$ and at most one write_sync control state $w_{i}$ belong to the reachable set $R_{i}(k)$. Thus, at depth $d, r_{i}$ (or $w_{i}$ ) block is paired with at most $(n \cdot d) w_{j}$ (or $r_{j}$ ) blocks. Since there are $n$ threads, we have at most $\left(n^{2} \cdot d\right)$ pairs. Thus, at depth $d$, the number of pair-wise constraints added, and variables introduced are $O(d)$, and number of non-pair constraints added is $O(1)$. Overall, the size of constraints and variables added up to depth $d$ is $O\left(d^{2}\right)$. Thus, the concurrency constraints grow quadratically with unrolling in the worst case.

For $X$ memory accesses up to depth $d$, the size complexity can be shown to be $O\left(X^{2}\right)$. To compare, the previous approaches [23,24], incur a cubic cost, i.e., $O\left(X^{3}\right)$, for a given memory model and a test program. $\square$.

\section{Removal of Redundant Concurrency Constraints}

We use various static analyses to reduce the number of context switches to consider. We identify and remove redundant concurrency constraints corresponding to them as follows:

- CFG transformation (PB): We use path/loop balancing transformations [21] on each thread model independently to obtain a reduced set of statically reachable blocks in CSR. This reduces concurrency and unrolled transition constraints. 
- Lockset-based analysis (MTX): We determine statically pair-wise unreachability of read_sync and write_sync control states using lockset $[9,10,14]$ analysis. For such pairs of read_sync and write_sync control states that are mutually exclusive (e.g., due to matching locks/unlocks), we do not add pair-wise constraints as the concurrency semantics forbids context-switching between those thread states.

- Context-sensitive CSR (CXT): In our modeling, we handle non-recursive procedures by creating a single copy and using extra variables to encode the call/return sites. (Recursive procedures are inlined upto some user-chosen depth). However, not inlining a procedure can cause false loops in the CFG of each thread, due to unmatched calls/returns of a procedure. We avoid saturation in CSR due to false loops in CSR by determining reachability in a context-sensitive manner, i.e., by matching the call/return sites for each procedure call. We observe in our experimental results that such analysis gives a dramatically reduced set of reachable read_sync and write_sync control states, and hence, a reduction in the set of pair-wise constraints added.

Discussion: The above mentioned static analysis techniques are not required to be precise as the imprecision does not affect completeness and soundness (Theorem 1) and size complexity of the overall analysis. However, less precise (but conservative) static analysis may result in less simplification and thereby poorer BMC performance. Furthermore, we can utilize transactions and/or partial order reductions $[8,14]$, to eliminate some pair-wise concurrency constraints.

\section{Experiments}

We experimented on the Daisy file system [31], a public benchmark used to evaluate and compare various concurrent verification techniques for concurrent threads communicating with shared memory and locks/unlocks. It is a 1 KLOC Java implementation of a representative file system, where each file is allocated a unique inode that stores the file parameters, and a unique block which stores data. Each access to a file, inode or block is guarded by a dedicated lock. Since a thread does not guarantee exclusive operations on byte access, potential race conditions exist. This system has some known data races. For our experiments, we used a $C$ version of the code [14] with a two-threaded concurrent system.

We conducted our experiments on an SMT-based BMC framework similar to [21]. We used the yices-1.0 [32] SMT solver at the back-end. We compared our lazy modeling with an eager modeling. In eager modeling approach, we add the pair-wise constraints in the model itself between the states with shared access, that also have waitcycles. We applied BMC simplification using CSR as discussed in Section 2.3 for all cases, referred to as the baseline strategy. We then combined this baseline strategy with other static analysis techniques such as path balancing/loop CFG transformations (PB) on CFG [21], context-sensitive analysis (CXT), and lockset analysis (MTX) $[9,10,14]$. We conducted controlled experiments with various combinations of these techniques.

\subsection{Comparing BMC results}

We now compare the performance of SMT-based BMC on detecting multiple data races, on both eager/lazy models, in various combinations of strategies. Note, multiple race detection is an optional feature. We detect multiple data races incrementally, by adding a blocking clause corresponding to the token passing events seen in the last witness trace to the satisfiable BMC instance, and then continuing the search. We conducted our experiments on a workstation with dual Intel $2.8 \mathrm{GHz}$ Xeon Processors with 4GB physical 
memory running Red Hat Linux 7.2, using a 6 hrs $(\approx 20 \mathrm{Ks})$ time limit and unroll bound limit of 300 for each BMC run. The results are summarized in Table 4(a). Column 1, shows the modeling approach (eager/lazy); Columns 2-6 show BMC results for various combinations of static analysis methods. Each data point $(d: t, m)$ corresponds to a performance summary of BMC up to depth $d$, with $t$ and $m$ representing the cumulative run time and memory used, respectively. Note, cumulative time includes the solve time incurred in the previous depths for the same run. We show a selected few data points for comparison. Specifically, Column 2 shows data for CSR with no PB and no CXT; Column 3 shows data for CSR with PB and no CXT; Column 4 shows data for CSR with PB and CXT; Column 5 shows data for CSR with PB, CXT and MTX; and Column 6 shows data for CSR with PB and MTX, but no CXT. For eager modeling, due to noninlining of procedure calls, we did not obtain any useful lockset information to reduce the constraints statically, and therefore, results in the columns (CSR+PB+CXT+MTX) and $(\mathrm{CSR}+\mathrm{PB}+\mathrm{MTX})$ are the same as $(\mathrm{CSR}+\mathrm{PB}+\mathrm{CXT})$ and $(\mathrm{CSR}+\mathrm{PB})$, respectively. As an example, consider BMC at unroll depth 64. BMC on eager model with CSR times out (TO) requiring $66 \mathrm{Mb}$, while on lazy model with CSR it takes $26 \mathrm{~s}$ and 39Mb.

In general, PB and CXT help BMC go deeper in both the eager and lazy models. However, CXT has a pronounced effect on the BMC performance. We also observed that the lockset analysis helps in improving the BMC performance, but not significantly. $\mathrm{BMC}$ on eager model, in general, does not go very deep, and times out in all cases without detecting any data races. In contrast, $\mathrm{BMC}$ on lazy model with $(\mathrm{CSR}+\mathrm{PB}+\mathrm{CXT})$ or $(\mathrm{CSR}+\mathrm{PB}+\mathrm{CXT}+\mathrm{MTX})$ is able to find 50 data races in a single $\mathrm{BMC}$ run. Note, less precise static analysis $\mathrm{CSR}$ and $\mathrm{CSR}+\mathrm{PB}$ show poorer performance compared to $\mathrm{CSR}+\mathrm{PB}+\mathrm{CXT}$ and $\mathrm{CSR}+\mathrm{PB}+\mathrm{CXT}+\mathrm{MTX}$.

In Table 4(b), we provide details of BMC performance on lazy models using CSR+PB+CXT+MTX on the first five data races. Column 1 shows the data races listed in the order of detection; Columns 2-4 show the BMC depth, cumulative time, and memory used, respectively. Column 5 shows the context-switches in the trace, each denoted as $\left(P_{i}: k_{i}, l_{i}\right) \rightarrow\left(P_{j}\right.$ : $k_{j}, l_{j}$ ) where model $P_{i}$ executes uninterrupted from depth $k_{i}$ to $l_{i}$, and then switches the context to $P_{j}$ at depth $k_{j}$. The Daisy example intentionally included many data races as a benchmark. Each race reported here corresponds to a unique set of context-switches in the witness trace. As an example, the first data race is detected at depth 143 taking $12 \mathrm{~s}$ and $10 \mathrm{Mb}$. There are 3 context switches: a $P_{1}$ run from depth 0 to 127 , followed by a $P_{2}$ run from depth 0 to 127 , followed by another $P_{1}$ run from 128 to 142 , followed by a data race detection when $P_{2}$ accesses the same variable at depth 128 . Note, the length of the trace is $271(=143+128)$.

\subsection{Comparison with related work}

In a related effort [14], two write-write data races were detected for the same benchmark, i.e. Daisy file system [31], using $1283 \mathrm{~s}$ and $122 \mathrm{Mb}$, and $5925 \mathrm{~s}$ and $902 \mathrm{Mb}$, respectively. Note, our eager modeling (used in experiments) differs from them mainly in the back-end solver, i.e., SMT vs SAT, and in use of static reduction methods, which was the crux of their approach. We believe that the orders of magnitude improvement in BMC performance (as reported in Table 4(b)), are attributed mainly to our lazy modeling paradigm that facilitates dramatic size-reduction of BMC instances.

In contrast to a closely related work TCMBC [15], we do not bound the number of context switches. Further, we add constraints lazily and incrementally during BMC unrolling. TCBMC, built over CBMC [33], translates concurrent $\mathrm{C}$ threads into static single assignment (SSA) form, and adds constraints for a bounded number of contextswitches for a bounded depth. TCMBC approach requires full inlining of functions and 
unwinding of loops like CBMC. This CBMC-based approach, therefore, is not scalable to large piece of code or code with reactive behavior, as shown previously [29].

We also contrast our work with $[23,24]$, where weaker memory models are considered. However, these approaches only check given test programs. Further, the number of concurrency constraints they add are cubic [24] in the number of shared accesses, while we add a quadratic number of constraints.

Table 4. SMT-based BMC (a) Comparison Results (b) Sample Witness Traces

(a) Comparing SMT-based BMC on Lazy/Eager Models

\begin{tabular}{|c|c|c|c|c|c|}
\hline \multirow{3}{*}{ Model } & \multicolumn{5}{|c|}{ Static Analysis Strategies } \\
\hline & $\begin{array}{l}\text { CSR } \\
\text { (1) }\end{array}$ & $\begin{array}{l}\mathrm{CSR}+\mathrm{PB} \\
(2)\end{array}$ & $\begin{array}{l}\mathrm{CSR}+\mathrm{PB} \\
+\mathrm{CXT}(3)\end{array}$ & $\begin{array}{c}\text { CSR+PB } \\
+\mathrm{CXT}+\mathrm{MTX}(4)\end{array}$ & $\begin{array}{l}\text { CSR+PB } \\
+\operatorname{MTX}(5)\end{array}$ \\
\hline & \multicolumn{5}{|c|}{$d: t, m$ with $d \equiv$ BMC Depth, $t \equiv$ Cum. Time(s), $m$} \\
\hline \multirow[t]{3}{*}{$\overline{\text { Eager }}$} & 64: TO,66 & 64: 132,21 & $64: 10,14$ & \multirow{3}{*}{$\begin{array}{c}\text { same as } \\
\text { (3) }\end{array}$} & \multirow{3}{*}{$\begin{array}{c}\text { same as } \\
\text { (2) }\end{array}$} \\
\hline & & $95: T 0,59$ & $95: 2 \mathrm{~K}, 31$ & & \\
\hline & & & $124: T 0,49$ & & \\
\hline race? & $\mathrm{N}$ & $\mathrm{N}$ & $\mathrm{N}$ & $\mathrm{N}$ & $\mathrm{N}$ \\
\hline \multirow[t]{5}{*}{ Lazy } & $64: 26,39$ & $64: 6,10$ & $64: 2,6$ & $64: 1,6$ & $64: 3,10$ \\
\hline & $73: 8 \mathrm{~K}, 101$ & $95: 35,10$ & $95: 5,8$ & $95: 4,8$ & $95: 17,28$ \\
\hline & \multirow{3}{*}{$\begin{array}{l}\text { Yices } \\
\text { aborted }\end{array}$} & 118: TO,114 & $118: 8,11$ & $118: 8,11$ & 118: $15 \mathrm{~K}, 108$ \\
\hline & & & $124: 16,10$ & $124: 9,10$ & 119: T0,112 \\
\hline & & & $287: 2.7 \mathrm{~K}, 34$ & $287: 2.4 \mathrm{~K}, 32$ & \\
\hline ace? & $\mathrm{N}$ & $\mathrm{N}$ & 50 races & 50 races & $\mathrm{N}$ \\
\hline
\end{tabular}

Note: $\mathrm{N} \equiv$ No Race Detected, ${ }^{*} \equiv$ Yices Aborted, $\mathrm{TO} \equiv$ Time Out (b) First 5 Data Race Traces using $\mathrm{CSR}+\mathrm{PB}+\mathrm{CXT}+\mathrm{MTX}$ on lazy model

\begin{tabular}{|c|c|c|c|c|}
\hline BMC & Time & \#em & $\begin{array}{c}\left(P_{i}: k_{i}, l_{i}\right) \\
\rightarrow\left(P_{j}: k_{j}, l_{j}\right) \\
P_{i} \text { executes from depths } \\
k_{i} \text { to } l_{i} \text { uninterrupted } \\
\text { and context-switches } \\
\text { to } P_{j} \text { at depth } k_{j} .\end{array}$ \\
\hline 1 & 143 & 12 & 10 & $\begin{array}{l}(1: 0,127) \rightarrow(2: 0,127) \rightarrow \\
(1: 128,142) \rightarrow(2: 128,-)\end{array}$ \\
\hline 2 & 174 & 25 & 13 & $\begin{array}{c}(1: 0,127) \rightarrow(2: 0,127) \rightarrow \\
(1: 128,173) \rightarrow(2: 128,-)\end{array}$ \\
\hline 3 & 180 & 30 & 15 & $\begin{array}{c}(1: 0,14) \rightarrow(2: 0,179) \\
\longrightarrow(1: 15,-)\end{array}$ \\
\hline 4 & 211 & 96 & 17 & $\begin{array}{c}(1: 0,127) \rightarrow(2: 0,158) \rightarrow \\
(1: 128,158) \rightarrow(2: 159,210) \\
\longrightarrow(1: 159,-)\end{array}$ \\
\hline 5 & 211 & 99 & 18 & $\begin{array}{c}(1: 0,127) \rightarrow(2: 0,210) \\
\rightarrow(1: 128,-)\end{array}$ \\
\hline
\end{tabular}

\section{Conclusions and Future Work}

We described a novel lazy modeling paradigm for shared memory multi-threaded concurrent systems, that is more suitable for BMC compared to synchronous modeling of interleaving semantics proposed previously. Such direct modeling of concurrency semantics in BMC is geared toward reducing the size of BMC instances, and thereby, improving the performance of BMC for deeper analysis. We add concurrency constraints lazily, incrementally and on-the-fly during BMC unrolling that preserve the concurrency semantics up to the bounded depth. We demonstrated several benefits of such an approach without incurring the cost of modeling interleaving semantics. By avoiding wait-cycles, our modeling allows greater scope for reduction in size of a BMC instance.. In addition, we use various static analyses to reduce the number of contextswitches to consider, which further reduces the size of the constraints. We demonstrated the efficacy of our approach on a complex example.

In future, we would like to combine partial-order reduction methods $[8,14]$, add deadlock detection, and analyze weaker memory models.

\section{References}

1. S. V. Adve and K. Gharachorloo. Shared memory consistency models: A tutorial. IEEE Computer, 1996.

2. L. Lamport. How to make multiprocessor computer that correctly executes multiprocess programs. IEEE Transactions on Computers, 1979.

3. A. Biere, A. Cimatti, E. M. Clarke, and Y. Zhu. Symbolic model checking without BDDs. In Proc. of TACAS, 1999.

4. Kenneth L. McMillan. Symbolic Model Checking. Kluwer Academic Publishers, 1993.

5. G. Ramalingam. Context sensitive synchronization sensitive analysis is undecidable. In ACM Transactions on Programming Languages and Systems, 2000. 
6. P. Godefroid. Model checking for programming languages using verisoft. In Proc. of POPL, 1997.

7. T. Andrews, S. Qadeer, S. K. Rajamani, J. Rehof, and Y. Xie. ZING: Exploiting program structure for model checking concurrent software. In Proc. of CONCUR, 2004.

8. P. Godefroid. Partial-order Methods for the Verification of Concurrent Systems: An Approach to the State-explosion Problem. PhD thesis, 1995.

9. C. Flanagan and S. Qadeer. Transactions for software model checking. In Proc. of TACAS, 2003.

10. S. D. Stoller. Model-checking multi-threaded distributed Java programs. Journal on STTT, 2002.

11. S. D. Stoller and E. Cohen. Optimistic synchronization-based state-space reduction. In Proc. of TACAS, 2003.

12. V. Levin, R. Palmer, S. Qadeer, and S. K. Rajamani. Sound transaction-based reduction without cycle detection. In Proc. of SPIN Workshop, 2003.

13. R. Alur, R. K. Brayton, T. A. Henzinger, S. Qadeer, and S. K. Rajamani. Partial-order reduction in symbolic state space exploration. In Proc. of CAV, pages 340-351, 1997.

14. V. Kahlon, A. Gupta, and N. Sinha. Symbolic model checking of concurrent programs using partial orders and on-the-fly transactions. In Proc. of CAV, 2006.

15. I. Rabinovitz and O. Grumberg. Bounded model checking of concurrent programs. In Proc. of $C A V, 2005$.

16. F. Lerda, N. Sinha, and M. Theobald. Symbolic model checking of software. In Electronic Notes Theoretical Computer Science, 2003.

17. S. Qadeer and J. Rehof. Context-bounded model checking of concurrent software. In Proc. of TACAS, 2005.

18. O. Grumberg, F. Lerda, O. Strichman, and M. Theobald. Proof-guided UnderapproximationWidening for Multi-process Sytems. In Proc. of POPL, 2005.

19. B. Cook, D. Kroening, and N. Sharygina. Symbolic Model Checking for Asynchronous Boolean Programs. In Proc. of SPIN Workshop, 2005.

20. B. Dutertre and L. de Moura. A fast linear-arithmetic solver for DPLL(T). In Proc. of CAV, 2006.

21. M. K. Ganai and A. Gupta. Accelerating high-level bounded model checking. In Proc. of ICCAD, 2006

22. S. V. Adve, M. D. Hill, B. P. Miller, and R. H. B. Netzer. Detecting data races on weak memory systems. In Proc. of ISCA, 1991.

23. Y. Yang, G. Gopalakrishnan, and G. Lindstrom. Memory-model-sensitive data race analysis. In Proc. of ICFEM, 2004.

24. S. Burckhardt, R. Alur, and M. M. K. Martin. CheckFence: Checking consistency of concurrent data types on relaxed memory models. In Proc. of PLDI, 2007.

25. Y. Yang, G. Gopalakrishnan, G. Lindstrom, and K. Slind. Nemos: A framework for axiomatic and executable specifications of memory consistency models. In Proc. of IPDPS, 2004.

26. Jan Wen Voung, Ranjit Jhala, and Sorin Lerner. Relay: static race detection on millions of lines of code. In ESEC/SIGSOFT FSE, pages 205-214, 2007.

27. Mayur Naik and Alex Aiken. Conditional must not aliasing for static race detection. In Proc. of POPL, pages 327-338, 2007.

28. L. Lamport. Time, clocks, and the ordering of events in a distributed system. Communications of the ACM, 1978.

29. F. Ivančić, Z. Yang, M. K. Ganai, A. Gupta, and P. Ashar. Efficient SAT-based Bounded Model Checking for Software Verification. In Proceedings of ISOLA, 2004.

30. M. K. Ganai and A. Gupta. Completeness in SMT-based BMC for software programs. In Proc. of DATE, 2008.

31. Joint CAV/ISSTA Special Event. Specification, Verification, and Testing of Concurrent Software. http://research.microsoft.com/ quadeer/cav-issta.htm, 2004.

32. SRI. Yices: An SMT solver. http://fm.csl.sri.com/yices.

33. D. Kroening, E. Clarke, and K. Yorav. Behavioral consistency of $\mathrm{C}$ and verilog programs using bounded model checking. In Proc. of DAC, 2003. 
$\star \star \star$ The appendix should not be considered as a part of the submission. $* \star \star$

\section{Appendix: Proofs}

\section{A Proof of Correctness}

We present the proof of soundness and completeness of our modeling approach. We start with a few definitions and notations.

Let there be $n$ thread models with $m$ shared variables. For each model $L M_{i}$, let $c_{i}^{k}$ denote its local state at depth $k$, and $P_{i}^{0, D}$ denote the set of finite sequences of its local state transitions, $c_{i}^{0, d(i)} \equiv c_{i}^{0}, \cdots, c_{i}^{d(i)}$, allowed by the transition relation and concurrency modeling constraints added up to $D$ for some $d(i) \leq D$, respectively. As a note, each model $L M_{i}$ has different $d(i)$. To simplify notation, we use $d$ instead of $d(i)$ without changing its meaning in the following.

Let the pair $\left(c_{i}^{k}, c_{i}^{k+1}\right)$ denote the local state transition between the corresponding states. Since the model $L M_{i}$ does not have self-loops, there is either a transition such that $c_{i}^{k+1} \neq c_{i}^{k}$, or the associated assume constraint (Eqn 12-13) does not hold. In the latter case, transitions from $c_{i}^{k}$ are terminated, i.e., $k=d$. Let $g_{i 1}^{k}, \cdots, g_{i m}^{k}, T K_{i}^{k}$, $C S_{i 1}^{k} \cdots C S_{i n}^{k}$ represent current state values of the localized shared variables of the model $L M_{i}$ in state $c_{i}^{k}$. Let $c_{i}^{k, l}$ denote the subsequence $c_{i}^{k}, \cdots, c_{i}^{l}$ of $c_{i}^{0, d}$.

Definition 1 A subsequence $c_{i}^{k, l}$ is called an initial subsequence (IS) iff the following hold

$-k=0, l<d$

- $\forall_{0 \leq h \leq l} T K_{i}^{h}=0, T K_{i}^{l+1}=1$

Note, each $c_{i}^{0, d}$ has at most one $I S$.

Definition 2 A subsequence $c_{i}^{k, l}$ is called an uninterrupted subsequence (US) iff the following holds

- $\exists_{k \leq h \leq l} T K_{i}^{k}=\cdots=T K_{i}^{h}=1, T K_{i}^{h+1}=\cdots T K_{i}^{l}=0$

$-T K_{i}^{k-1}=0$, if $k \neq 0$

$-T K_{i}^{l+1}=1$, if $l \neq d$

A $U S$, denoted as $c_{i}^{k, h, l}$, is said to be complete iff $h<l$; otherwise, it is said to be incomplete, i.e., $h=l$. Note, an incomplete $U S$ has $T K_{i}^{k}=1$ in all states in the subsequence.

Using Definitions 1 and 2, we chop the sequence $c_{i}^{0, d}$ into subsequences of $I S$ and US. We can easily show by virtue of construction that for a subsequence $c_{i}^{k, h, l}$ with $l \neq d$, there is another subsequence $c_{i}^{l+1, h^{\prime}, l^{\prime}}$. These subsequences can be concatenated (denoted by ::), i.e., $c_{i}^{k, h, l}:: c_{i}^{l+1, h^{\prime}, l^{\prime}}$, to obtain the sequence $c_{i}^{0, d}$. We say these subsequences follow concatenation order. 
Example: We illustrate above definitions using an example shown in Figure 4. Let there be three threads $P_{1}, P_{2}$ and $P_{3}$ with local sequences $c_{1}^{0,8}, c_{2}^{0,8}$, and $c_{3}^{0,6}$, respectively. Let each box represent the local state $c_{i}^{k}$ of the thread $P_{i}$ at step $k$. The value $1 / 0$ inside the shaded/unshaded box denotes the token value as seen at the thread state. The subsequences $U S$ are shown by bold rectangle, and $I S$ are shown with dash outlines. Note, all US subsequences are complete except one, i.e., $c_{2}^{6,8,8}$ indicating $P_{2}$ is the current holder of the token. We will show later that given these subsequences, there exists a unique total order on the subsequences, denoted as IUS that satisfies the concurrency constraints up to depth 8 .

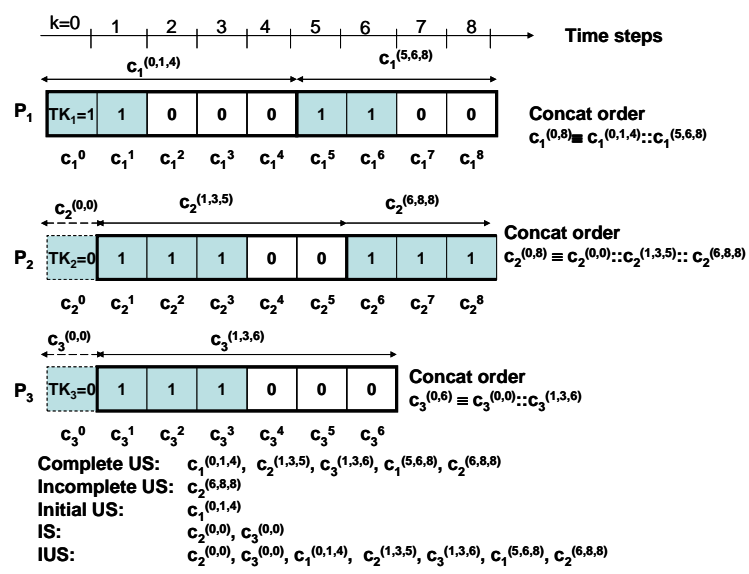

Fig. 4. Ilustrating various definition of subsequences.

Definition 3 A local sequence $c_{i}^{0, d}$ can be one of the following types I, II, or III depending on the existence of IS and US in the sequence.

- Type I: IS does not exist and at least one US exists. One can concatenate multiple US such that $c_{i}^{0, d} \equiv c_{i}^{0, h, l}:: c_{i}^{l+1, h^{\prime}, l^{\prime}}:: \cdots:: c_{i}^{l^{\prime \prime}, h^{\prime \prime}, d}$.

- Type II: IS exists (implying existence of at least one US) such that one can concatenate IS and US to obtain $c_{i}^{0, d}$, i.e., $c_{i}^{0, d} \equiv c_{i}^{0, l}:: c_{i}^{l+1, h^{\prime}, l^{\prime}}:: \cdots:: c_{i}^{l^{\prime \prime}, h^{\prime \prime}, d}$.

- Type III: Neither an IS nor a US exists for the local sequence.

One can show existence of such concatenations for types I and II using the definition of $U S$. If $I S$ exists, there exists at least one $U S$ in a sequence $c_{i}^{0, d}$ by construction.

Example: In Figure $4, c_{1}^{0,8} \equiv c_{1}^{0,1,4}:: c_{1}^{5,6,8}$ is a type $I$ sequence and $c_{2}^{0,8} \equiv c_{2}^{0,0}::$ $c_{2}^{1,3,5}:: c_{2}^{6,8,8}$ and $c_{1}^{0,6} \equiv c_{3}^{0,0}:: c_{3}^{1,3,6}$ are type II sequences.

Definition 4 We say an ordered pair $\left(c_{j}^{k^{\prime}, h^{\prime}, l^{\prime}}, c_{i}^{k, h, l}\right)$ of US from different threads is valid iff $R W_{i j}^{(k-1) h^{\prime}}=1$, for $k>0$, and $h^{\prime}<l^{\prime}$, i.e., token passing occurs. 
We have following program order property on each local sequence $c_{i}^{0, d}$.

Lemma 1. Each local sequence $c_{i}^{0, d}$ follows program order.

Proof: Note, the annotations (Section 5.1) do not change the relative order of the thread statements. Further, if all Read-Write Synchronization Enabling Constraint are disabled, i.e., $\forall_{j \neq i, 0 \leq h \leq d} R W_{i j}^{k h}=0$, No Sync Update Constraint (Eqn 8-11) ensures that each thread model $L M_{i}$ behaves identical to $M_{i}$. Therefore, the program order of memory access is also preserved in these sequences.

Lemma 2. Type III sequences, by Definition 3, cannot affect global state, and therefore, can be ignored while analyzing global state.

Proof: Recall, type III sequence corresponds to a local execution from the initial state, where the write to shared variables have not been committed. Thus, such sequences cannot affect the global state, and therefore, can be ignored. Note, that the constraint Single Control State Reachability Property Constraints (Eqn 14) requires that $T K_{i}=1$ when the local control state $a$ is reachable, and therefore, witnesses are necessarily either type I or III sequences.

Lemma 3. Concatenation order of type I and II sequences follows program order and is unique for a local sequence.

Proof: Follows from the definitions of type $I$ and $I I$ sequences and Lemma 1.

Lemma 4. For a valid ordered pair $\left(c_{j}^{k^{\prime}, h^{\prime}, l^{\prime}}, c_{i}^{k, h, l}\right)$, the following hold:

- $\forall_{q \neq i}^{n} C S_{q i}^{k}=C S_{q j}^{h^{\prime}}$

- $C S_{i i}^{k}=C S_{i j}^{h^{\prime}}+1$

- $\forall{ }_{p=1}^{m} g_{p i}^{k}=g_{p j}^{h^{\prime}}$

Proof: For a valid ordered pair, $R W_{i j}^{(k-1) h^{\prime}}=1$ holds. Hence, from Eqn $1, C S_{i i}^{k-1}=$ $C S_{i j}^{h^{\prime}}$. The proof follows from Read-Write Synchronization Update Constraints (Eqn 4 and 6).

Lemma 5. For an IS or US $c_{i}^{k, h, l}$ the following hold:

$$
\text { - } \forall_{q=1}^{n} C S_{q i}^{k}=C S_{q i}^{l}
$$

Proof: Since in $I S$ and $U S$, there is no transition of token value from 0 to $1, R C_{i}^{k}=$ $\cdots=R C_{i}^{l}=0$. Applying No Sync Update Constraint (Eqn 10), the proof follows.

Lemma 6. Given a concatenation, $c_{i}^{k, h, l}:: \cdots:: c_{i}^{k^{\prime}, h^{\prime}, l^{\prime}}$, the following hold:

- $C S_{i i}^{k} \leq C S_{i i}^{k^{\prime}}$ 
Proof: Note, $k<k^{\prime}$ as concatenation order is in program order (Lemma 3). Further, since the only update constraints (Eqn 6 and 10) do not decrease $C S_{i i}^{k^{\prime}}$ for $k^{\prime}>k$, the proof follows.

Lemma 7. There is a unique US $c_{i}^{k, h, l}$ with $k=0$ for some $i$.

Proof: It follows from the Single Token Constraint (Eqn 15), that allows only one model, say $i$, to have token initially.

Lemma 8. For an $U S c_{i}^{k, h, l}$ with $k \neq 0$, there exists a unique complete $U S c_{j}^{k^{\prime}, h^{\prime}, l^{\prime}}$ with $h^{\prime}<l^{\prime}$ such that the ordered pair $\left(c_{j}^{k^{\prime}, h^{\prime}, l^{\prime}}, c_{i}^{k, h, l}\right)$ is valid.

Proof: From the Definition $2, c_{i}^{k, h, l}$ with $k \neq 0$ implies $T K_{i}^{k-1} \neq T K_{i}^{k}$; which in turn implies $R C_{i}^{k-1} \neq 0$, using Eqn 9. W.1.o.g. (Without loss of generality), let $R C_{i}^{k-1}=a_{j}^{h^{\prime}}$, which with Eqn 2 implies $R W_{i j}^{(k-1) h^{\prime}}=1$. Thus, as per Definition 4, the ordered pair $\left(c_{j}^{k^{\prime}, h^{\prime}, l^{\prime}}, c_{i}^{k, h, l}\right)$ is valid.

Lemma 9. For a complete US $c_{j}^{\left(k^{\prime}, h^{\prime}, l^{\prime}\right)}$ with $h^{\prime}<l^{\prime}$, there exists a unique US $c_{i}^{k, h, l}$ such that the ordered pair $\left(c_{j}^{k^{\prime}, h^{\prime}, l^{\prime}}, c_{i}^{k, h, l}\right)$ is valid.

Proof: From the Definition $2, c_{j}^{k^{\prime}, h^{\prime}, l^{\prime}}$ with $h^{\prime}<l^{\prime}$ implies $T K_{j}^{h^{\prime}+1}=0$ and $T K_{j}^{h^{\prime}}=1$; which in turn implies $W C_{j}^{h^{\prime}} \neq 0$, using Eqn 11. W.l.o.g., let $W C_{j}^{h^{\prime}}=a_{i}^{k}$, which with Eqn 3 implies $R W_{i j}^{(k-1) h^{\prime}}=1$. Thus, as per Definition 4, the ordered pair $\left(c_{j}^{k^{\prime}, h^{\prime}, l^{\prime}}, c_{i}^{k, h, l}\right)$ is valid.

Lemma 10. A global sequence of US (from different threads) can be constructed so that every consecutive pair of US is a valid ordered pair as per Definition 4. Further, such a sequence starts with a unique $c_{i}^{0, h, l}$ for some $i$.

Proof: As per Lemma 7, there exists a unique $U S c_{i}^{0, h, l}$. We start a sequence with the unique $U S$. If $h=l$, we stop; otherwise using Lemma 9, we obtain a next $U S$ in the sequence, say, $c_{j}^{k^{\prime}, h^{\prime}, l^{\prime}}$ and continue until $h^{\prime}=l^{\prime}$. Note, we stop at an incomplete US as per Definition 2.

In the following Lemma 11- 13, we prove that the global sequence of US, so constructed, has the following properties: finite, cycle-free, unique, and a total orderedinterleaving sequence of US in the program order.

Lemma 11. The global sequence of US (from different thread) is finite, has no cycle and always ends with an incomplete US. 
Proof: As the number of $U S$ is finite for a local sequence $c_{i}^{0, d}$, and number of threads are finite, i.e., $n$, we only have to show that there is no cycle in the sequence of US. To prove by contradiction, we assume there exists a cycle, i.e., $c_{i}^{k, h, l}, \cdots, c_{j}^{k^{\prime}, h^{\prime}, l^{\prime}}, c_{i}^{k, h, l}$. W.l.o.g., let there be no nested cycle in this cycle. Applying Lemma 4 on consecutive $U S$ in the sequence $c_{i}^{k, h, l}, \cdots, c_{j}^{k^{\prime}, h^{\prime}, l^{\prime}}$, one can show that $C S_{i i}^{k} \leq C S_{i j}^{k^{\prime}}$. Again, applying Lemma 4 and 5 on $\left(c_{j}^{k^{\prime}}, h^{\prime}, l^{\prime}, c_{i}^{k, h, l}\right)$, we show that $C S_{i j}^{k^{\prime}}=C S_{i j}^{l^{\prime}}$ and $C S_{i j}^{l^{\prime}}+1=C S_{i i}^{k}$. Thus, we obtain a contradiction $C S_{i i}^{k}<C S_{i i}^{k}$. Therefore, we conclude that the sequence is finite with no cycle and always ends with an incomplete $U S$ as per construction using Lemma 10.

Lemma 12. The global sequence of US is unique.

Proof: We show this by induction on the subsequence length.

- Basis: Sequence with one US.

This is trivially true as there is a unique $U S c_{i}^{0, h, l}$ as per Lemma 7.

- Induction: Given a unique global subsequence of $U S$ of length $t$ starting with a unique $U S$ and ending with a complete $U S$, we show that the subsequence of length $t+1$ is also unique. Note, if the last $U S$ is incomplete, the induction step is trivially true since there can be only one incomplete US in a global sequence of US.

Let the $t^{t h} U S$ in the global sequence be $c_{j}^{k^{\prime}, h^{\prime}, l^{\prime}}$. We use Lemma 9 to obtain a unique next $U S$. Thus, the subsequence of length $t+1$ is also unique.

Lemma 13. The global sequence of US is a total order-ed sequence and maintains program order.

Proof: We show that the global sequence includes all the local sequences of $U S$ in the concatenation order.

- Inclusion: To show total inclusion, we show a given $U S c_{i}^{k, h, l}$, is not left out, i.e., it is always included in the global sequence. If $k=0$, we are done trivially, as per Lemma 7 this $U S$ is unique, and the global sequence starts with this US (Lemma 10); otherwise, we use Lemma 8 repeatedly, finitely often as per Lemma 11, and obtain another $U S c_{j}^{k^{\prime}, h^{\prime}, l^{\prime}}$ preceding it, until $k^{\prime}=0$. As per Lemma 12 , there is a unique global sequence, and the sequence $c_{j}^{0, h^{\prime}, l^{\prime}} \cdots c_{i}^{k, h, l}$ is a subsequence of the global sequence.

- Concatenation order: We show this by contradiction. Given a concatenated pair of US $c_{i}^{k, h, l}:: c_{i}^{l+1, h^{\prime}, l^{\prime}}$, we assume that in the global sequence, we have a subsequence $c_{i}^{l+1, h^{\prime}, l^{\prime}} \ldots c_{i}^{k, h, l}$, where the pair does not appear in concatenation order. Applying Lemma 4 on consecutive $U S$ in the global sequence $c_{i}^{l+1, h^{\prime}, l^{\prime}}, \cdots, c_{i}^{k, h, l}$, one can show that $C S_{i i}^{l+1}<C S_{i i}^{k}$. However, using Lemma 6, we obtain, $C S_{i i}^{k} \leq$ $C S_{i i}^{l+1}$; a contradiction.

Thus, inclusion and concatenation order rules give a total interleaving of the local sequences of $U S$ and using Lemma 3 we also obtain that the global sequence of $U S$ maintains the program order. 
We construct a global sequence, denoted as $I U S$, by sequencing all $I S$ in some total order before the global sequence of $U S$.

Example: In Figure 4, the global sequence $c_{2}^{0,0}, c_{3}^{0,0}, c_{1}^{0,1,4}, c_{2}^{1,3,5}, c_{3}^{1,3,6}, c_{1}^{5,6,8}, c_{2}^{6,8,8}$ represents an IUS.

Now, we prove that IUS holds the sequential consistency and synchronization semantics.

Lemma 14. The sequence IUS maintains program order.

Proof: This follows trivially from Lemma 1 and 13.

Lemma 15. The sequence IUS has a total order of memory access.

Proof: As per Lemma 13, the global sequence of US is a total ordered sequence. Further, since we choose a total order on $I S$, the proof follows.

Lemma 16. Read value rule is maintained in IUS, i.e., a read access of a shared variable observes the effect of the last write access to the same variable in the sequence IUS.

Proof: In each US, Read Value Rule is observed due to program semantics as per Lemma 1. Between consecutive US (from different threads), Read Value Rule is maintained by Lemma 4. In each $I S$, and in the starting unique $U S$, the read of shared variable sees the initial state of the shared memory, which is the same for all thread models.

Lemma 17. Shared accesses by different threads in matched lock/unlock operations are mutually exclusive in IUS.

Proof: It is is sufficient to show that the mutually exclusive shared access does not occur twice in the IUS sequence with same lock variable being set to 1 without setting it to 0 in between.

W.l.o.g., assume a shared variable is accessed simultaneously in a state $a_{i}$ and $b_{j}$ that are mutually exclusive using lock $l k$ in the global sequence of IUS. Let $c_{i}^{k, h, l}$ denote a $U S$ such that $l k_{i}^{l}$ has value 1 . Let $c_{j}^{k^{\prime}, h^{\prime}, l^{\prime}}$ denote $U S$ such that for some $k^{\prime} \leq t \leq h^{\prime}$, $l k_{1, j}^{t+1}$ gets value 1 in control state $c_{j}^{t+1}$ due to an update from a lock control state $c_{j}^{t}$. Let $c_{i}^{k, h, l}$ occur before $c_{j}^{k^{\prime}, h^{\prime}, l^{\prime}}$ in the IUS. We further assume that there exists no other sequence corresponding to lock/unlock of lock $l k$. A necessary condition for exclusive violation to occur, is to show that there exists in the IUS a transition from lock acquisition control state, i.e., $c_{j}^{t}$ with $t<l^{\prime}$. Note, $l k_{j}^{k^{\prime}}=l k_{i}^{l}=1$ as Read Value Rule is maintained (Lemma 16), which the Mutual Exclusion Constraint (Eqn 12), $B_{c_{j}^{t}} \Longrightarrow\left(l k_{j}^{t}=0\right)$, there is a clear conflict. Hence, such a global sequence IUS does not exist where lock variable is set to 1 twice without setting it to 0 . Similarly, we can argue for unlock case, where lock is set to 0 twice without setting it to 1 .

We now present the following Theorem validating the soundness and completeness of our lazy modeling approach for BMC. 
Theorem 1 The lazy modeling constraints allow only those traces that respect the sequential consistency of memory model and synchronization schematics up to the bound $D$, i.e., our modeling is complete.

Further, if there exists witness for a reachability property, such that the global trace length is $\leq n \cdot D$ and each local trace length $\leq D$, there exists an equivalent trace allowed by our model, i.e., IUS corresponding to the witness trace. In other words, our modeling is sound in that it does not miss any witness up to these bounds.

Proof:

- Completeness: Recall, for a given BMC bound $D, P_{i}^{0, D}$ denotes the set of sequences of local state transitions up to the bound. Given a tuple of local state transitions $\left(c_{1}^{0, d(1)}, \cdots, c_{n}^{0, d(n)}\right) \in P_{1}^{0, D} \times \cdots \times P_{n}^{0, D}$, we construct a unique global sequence IUS, that capture the requirements of sequential consistency of memory model and synchronization semantics up to the bound $D$ (Lemmas 14-17). Thus, the IUS represents a valid trace in the concurrent system w.r.t sequential consistency and synchronization semantics.

- Soundness: We show that for a given witness trace that follows sequential consistency and synchronization semantics, there exists a tuple of local state transitions $\left(c_{1}^{0, d(1)}, \cdots, c_{n}^{0, d(n)}\right)$. Note, from this tuple we construct a unique IUS (using Lemma 10 and 12).

Let us take a total ordered $t^{t h}$-length memory trace $A_{i}^{0} \cdots A_{j}^{t}$ that is sequentially consistent and follows synchronization semantics and is a witness to the given reachability property, where $A_{j}^{t}$ represents a shared access by thread $P_{j}$ at $t^{t h}$ step. We chop this trace into subsequences of consecutive accesses of a thread $P_{i}$, i.e., $A_{i}^{k}, \cdots, A_{i}^{l}$. We then append a NOP operation $N_{i}^{l+1}$ after $A_{i}^{l}$. We obtain an equivalent $U S$ from each such subsequence by associating a token $T K_{i}$ such that $T K_{i}^{k}=\cdots=T K_{i}^{h}=1$, and $T K_{i}^{h+1}=\cdots=T K_{i}^{l+1}=0$, where we choose $h$ from one of the following:

- $h=k$, if there is no write in the subsequence

- $h=r$, if subsequence is not the last subsequence, and $A_{i}^{r}$ is the last write in the subsequence, $k \leq r \leq l$

- $h=l$, if the subsequence is the last sequence (and there is a write operation in the subsequence).

We then obtain a local sequence of $U S$ for each thread $P_{i}$, by taking the $U S$ corresponding to the thread $P_{i}$ and concatenate them in the same order as they appear in the witness. Note, each local sequence follows the program order (guaranteed by the witness), which can be represented by the tuple of local state transition $c_{1}^{0, d(1)}, \cdots, c_{n}^{0, d(n)}$. Note, each local sequence is a type I or II sequences by Definition 3 .

Comments: The maximum length of an IUS can be $n \cdot D$ with the restriction that each local sequence, combining $I S$ and $U S$, is of maximum length $D$. Note, the construction of IUS is only conceptual. Indeed, we use BMC to explore all such possible IUS to find a violation or a witness for a reachability property. 